\title{
Treatment of oat cell carcinoma of the lung : complete remissions, acceptable complications, and improved survival
}

\author{
F A GRECO, R L RICHARDSON, S F SCHULMAN, S STROUP, R K OLDHAM
}

British Medical fournal, 1978, 2, 10-11

\section{Summary and conclusions}

Oat cell lung cancer is a common disease which is usually disseminated by the time it is diagnosed. Treatment with cyclic combination chemotherapy (cyclophosphamide, doxorubicin, vincristine) administered concurrently with radiotherapy to the chest lesion and subsequent prophylactic brain irradiation was investigated in 36 patients with oat-cell carcinoma of the lung. Complete remissions occurred in 26 of the patients (15 of the 16 with limited-stage disease and 11 of the 20 with extensivestage disease). Symptomatic improvement occurred in all patients. Twelve of the 16 patients with limited disease remained well and free of disease for over a year. The results were equivalent to those of a similar though more intensive regimen, but the toxicity was much less (there were no treatment-related deaths). Transient granulocytopenia with the risk of infection was the most serious complication.

Survival and quality of life have been improved for all patients, particularly those with limited disease, who have previously responded poorly to treatment.

\section{Introduction}

Unlike other types of lung cancer, oat cell carcinomas, which represented $20^{\circ}{ }_{o}$ of all lung cancers in the USA in $1977,{ }^{1}$ spread early outside the thoracic cavity, ${ }^{2}$ resulting in rapid clinical deterioration. Past treatments have had little effect on the course of the disease, though remission has recently been reported after combination chemotherapy either alone or with radiotherapy ${ }^{3-5}$ : Johnson et al reported a complete remission rate of $95^{\circ}{ }^{4}$ after intensive chemotherapy with cyclophosphamide, doxorubicin, and vincristine given concurrently with chest and brain radiotherapy. But the morbidity ${ }^{5-7}$ and mortality ${ }^{8}\left(24^{\circ}{ }_{0}\right)$ on this regimen were excessive. We therefore modified the regimen and gave the drugs less often, used smaller doses of cyclophosphamide, and gave only one daily fraction of radiotherapy.

\section{Patients and methods}

Thirty-six consecutive untreated patients with biopsy-proved oat cell carcinoma of the lung were treated from January 1976 to January 1977. The 32 men and four women had a median age of 59 years (range 46-76 years). Staging evaluation before treatment included chest radiographs; fibreoptic bronchoscopy; radionuclide liver, bone, and brain scans; and bone marrow aspiration and biopsy. Patients

Divisions of Medical Oncology and Radiation Oncology, Vanderbilt University Medical Centre, Nashville, Tennessee, USA

F A GRECO, MD, assistant professor of medicine

R L RICHARDSON, MD, assistant professor of medicine

$S$ F SCHULMAN, AB, data manager

$S$ STROUP, MD, assistant professor of radiology

R K OLDHAM, MD, associate professor of medicine were designated as having limited-stage disease if the disease seemed to be limited to one hemithorax or the mediastinum or supraclavicular area and was encompassable by one radiation therapy port. Patients were designated as having extensive-stage disease if disease was also detected elsewhere. Sixteen patients had limited disease and 20 had extensive disease. Of those with extensive disease, 10 had positive findings on bone-marrow biopsy or aspirations, 12 had positive bone scans, two had subcutaneous metastases, and 10 had positive liver scans. Of the 16 patients with limited disease several had large mediastinal or lung masses, or both.

All patients with limited disease were ambulatory, but 13 had cough, chest pain, or shortness of breath, and eight had lost $10^{\circ}{ }^{\circ}$ or more of their body weight. By contrast, four of the 20 patients with extensive disease were bedridden and 10 were ambulatory only for a fraction of the day. Nineteen had disease-related symptoms and 15 of the 20 had lost $10^{\circ}{ }_{0}$ or more of their body weight.

Treatment-Radiotherapy to the affected lung, mediastinum, and supraclavicular areas was given in 10 300-rad fractions over two weeks (3000 rad). It was started concomitantly with intravenous combination chemotherapy with cyclophosphamide $\left(1000 \mathrm{mg} / \mathrm{m}^{2}\right)$, doxorubicin (40 $\left.\mathrm{mg} / \mathrm{m}^{2}\right)$, and vincristine $\left(1 \mathrm{mg} / \mathrm{m}^{2}\right)$. The chemotherapy was continued in the outpatient clinic every 21 days for six cycles. Drug doses were adjusted downwards or withheld temporarily if there was evidence of haematological toxicity from blood counts made on the day of treatment (see table). Eighteen of the 36 patients needed at least one dose adjustment or delay in treatment. After six cycles of chemotherapy most patients were placed on continued chemotherapy during remission with three monthly cycles of the investigational drugs hexamethylmelamine and etoposide (an epipodophyllotoxin).

Evaluation of response-Response to treatment was evaluated after two cycles of combination chemotherapy by physical examination and chest radiography and after six cycles by repeating the pretreatment staging studies. Fibreoptic bronchoscopy was repeated in 12 of the 16 patients with limited-stage disease. A complete remission was defined as a complete resolution of all previously detectable disease. In patients with extensive disease the bone scan result might remain positive but the other scans and bone marrow had to become normal. Chest radiographs had to show complete resolution of masses or only residual changes consistent with radiation effect. Partial remission was defined as a $50 \%$ or greater decrease in the perpendicular diameters of the measured tumour masses.

Dose adjustment schedule

\begin{tabular}{|c|c|c|c|c|}
\hline \multicolumn{2}{|c|}{ Blood counts } & \multicolumn{3}{|c|}{ Dose adjustment $(\%$ of full dose $)$} \\
\hline$\underset{\left(\times 10^{9} / 1\right)}{\text { WBC }}$ & $\begin{array}{l}\text { Platelets } \\
\left(\times 10^{9} / 1\right)\end{array}$ & Vincristine & $\begin{array}{c}\text { Cyclophospha- } \\
\text { mide }\end{array}$ & Doxorubicin \\
\hline $\begin{array}{l}>4 \\
3-4 \\
2-3 \\
<2\end{array}$ & $\begin{array}{c}>150 \\
125-150 \\
75-125 \\
<75\end{array}$ & $\begin{array}{l}100 \\
100 \\
100\end{array}$ & $\begin{array}{c}100 \\
75 \\
50 \\
\text { dd till following }\end{array}$ & $\begin{array}{r}100 \\
75 \\
50\end{array}$ \\
\hline
\end{tabular}

\section{Results}

RESPONSE RATE AND DURATION

All patients responded to treatment. Twenty-six $(72 \%)$ had complete remission. Eleven of the 20 patients $(55 \%)$ with extensive disease had complete remission; but eight of these 11 had stable or improved but persistently abnormal bone scans. The median duration of response was eight months (range 2-18 months). Fifteen of the 16 patients with limited disease obtained a complete remission $(94 \%)$. Repeat fibreoptic bronchoscopy was performed in 12 of these patients. All had patent airways; and biopsies, brushings, and washings of 
previously affected bronchi were negative for tumour. At the time of writing the median duration of response had not been reached in patients with limited-stage disease. Twelve of the 16 patients had been in complete remission for over a year (range 12-20 months).

Every patient with symptoms had the symptoms decreased or eliminated. Three of four non-ambulatory patients became ambulatory and all 10 of those who were ambulatory for only short periods of time became more ambulatory. Several patients began to gain weight during the eighth or ninth week of treatment and six out of eight patients with limited disease regained their previous body weight.

\section{SURVIVAL IN PATIENTS WITH LIMITED DISEASE}

Twelve of the 16 patients with limited disease were alive and free of clinical disease for over a year after the start of treatment (see figure) All were ambulatory with normal functional activity. Three of the four patients who died were among the first patients treated, and they developed cerebral metastasis as their first recurrence. The other patient was the sole partial responder and he died of progressive disease eight months after starting treatment. Because of the brain relapses in the initial patients and controlled studies that have shown the preventive value of prophylactic brain irradiation, ${ }^{911}$ all subsequent patients with limited disease were given prophylactic brain irradiation at a dose of 3000 rads over two weeks.

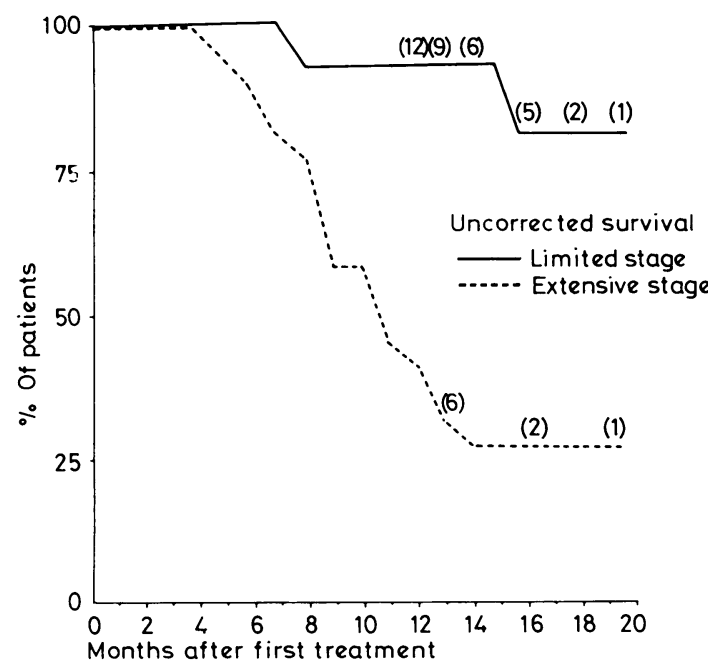

Acturial uncorrected survival for patients with limited-stage and extensive-stage disease. Numbers surviving at each time are shown in parentheses.

\section{SURVIVAL IN PATIENTS WITH EXTENSIVE DISEASE}

Patients with extensive disease did less well (see figure). Although they responded and improved symptomatically, all subsequently developed progressive disease. Only four were alive at the time of writing, and each had evidence of recurrent disease.

\section{TREATMENT TOXICITY AND COMPLICATIONS}

No patient died from complications associated with treatment. The most serious complication was transient neutropenia. Eight patients developed 10 episodes of fever requiring a stay in hospital during a total of 216 courses of chemotherapy. Three patients developed an infection, and all were successfully treated with antibiotics. The mean nadir neutrophil count was $0.9 \times 10^{9} / 1$ (range of $0.050-2 \cdot 0 \times 10^{9} / 1$ ). No patient had thrombocytopenic bleeding or needed platelet transfusion. The mean platelet nadir was $90 \times 10^{9} / 1$ (range of $25-150 \times 10^{9} / 1$ ).

All patients had transient nausea, and $60 \%$ had vomiting associated with chemotherapy but none had to be admitted to hospital. Transient alopecia was seen in all patients. Mild to moderate oesophagitis developed in 20 patients, but only two needed admission for brief periods while intravenous fluids were given. No patient developed recurrent oesophagitis after chemotherapy or oesophageal strictures. Erythema over irradiated skin occurred in all patients but only two developed a transient moist desquamation. No doxorubicin cardiotoxicity or acute pneumonitis induced by radiation or drugs was seen.

\section{Discussion}

Combined chemotherapy and radiotherapy produced a $72 \%$ complete remission rate in patients with oat cell carcinoma of the lung. The toxicity and complications of treatment were acceptable, and no patient died from them. The overall complete remission rate was equivalent to that produced by a similar though more intensive and toxic regimen. ${ }^{458}$

Complete remission and survival after treatment were closely related to the stage of disease. Fifty-five per cent of patients with extensive disease had complete remissions, though if complete normality of radionuclide bone scans is taken as the criterion of remission then only three of the 20 patients $\left(15^{\circ}\right.$ ) had a complete remission. These patients had a shorter period of symptomatic improvement and survival than those with limitedstage disease; and their tumour volume was larger and their physical condition and functional status were generally worse. Continued clinical investigation is necessary to improve the complete remission rate in patients with extensive-stage disease. Improvements in these patients can easily be applied to those with lesser tumour burdens (limited stage) with even greater expectations.

Fifteen of the 16 patients with limited-stage disease had a complete remission $(94 \%)$, and at the time of writing 12 had been well and free of detectable disease for over a year after the start of treatment. Recently, Einhorn, using a somewhat similar regimen, has observed five out of 19 patients with limited-stage disease $\left(26^{\circ}, 1\right)$ who were alive and disease free after 26 months. ${ }^{11}$ Patients with limited-stage disease have therefore experienced long "disease-free" periods on this treatment and are potentially cured. This possibility is important because patients with limited disease do poorly when treated with surgery, radiotherapy, or less effective chemotherapy. ${ }^{3}$

The treatment outlined is clearly effective in producing complete or partial remissions and reducing or eliminating the symptoms and signs of oat cell carcinoma for varying periods. Whether further treatment during remission can prolong useful survival is not yet known. The role of radiotherapy for the chest lesion is also unknown and must be investigated by comparative studies. Although patients benefit clinically from this treatment, the degree and length of improvement are certainly related to the stage of their disease and general condition. All patients improved symptomatically and some of those with limited disease may have had their disease eradicated.

Attention to therapeutic toxicity is necessary, particularly the risk of infection associated with neutropenia, but the benefits of this treatment far exceed the toxicity. Induction treatment is readily available and may now be viewed as similar to combination chemotherapy for patients with advanced breast cancer.

\section{References}

${ }^{1}$ Slidman, H, Silverberg, E, and Holleb, A, Cancer Statistics. New York, American Cancer Society, Professional Education Publications, 1976.

${ }^{2}$ Mountain, C F, Carr, D T, and Anderson, W A, American fournal of Roentgenology, Radium Therapy, and Nuclear Medicine, 1974, 120, 130.

${ }^{3}$ Bunn, P A, et al, Cancer Treatment Reports, 1977, 61, 333.

${ }^{4}$ Johnson, R E, Brereton, H D, and Kent, H C, Lancet, 1976, 2, 289.

${ }^{5}$ Kent, H C, Brereton, H D, and Johnson, R E, International fournal of Radiation Oncology Biology and Physics, 1977, 2, 427.

${ }^{6}$ Greco, F A, et al, Annals of Internal Medicine, 1976, 85, 294

${ }^{7}$ Merrill, J M, et al, Lancet, 1976, 1, 1105.

${ }^{8}$ Brereton, H D, Kent, H C, and Johnson, R E, Proceedings of the American Society of Clinical Oncology, 1977, 18, 275.

9 Tullah, M E, Maurer, L H, and Forrier, R J, Proceedings of the American Society of Clinical Oncology, 1977, 18, 268.

${ }^{10}$ Jackson, D V, et al, Proceedings of the American Society of Clinical Oncology, $1977,18,319$.

${ }^{11}$ Einhorn, L H, et al, Seminars in Oncology. In press. 